\title{
LIBROS ELECTRÓNICOS: OFERTA COMERCIAL Y REDES
} P2P

\author{
José-Antonio Cordón-García, Raquel Gómez-Díaz y Julio Alonso-Arévalo
}

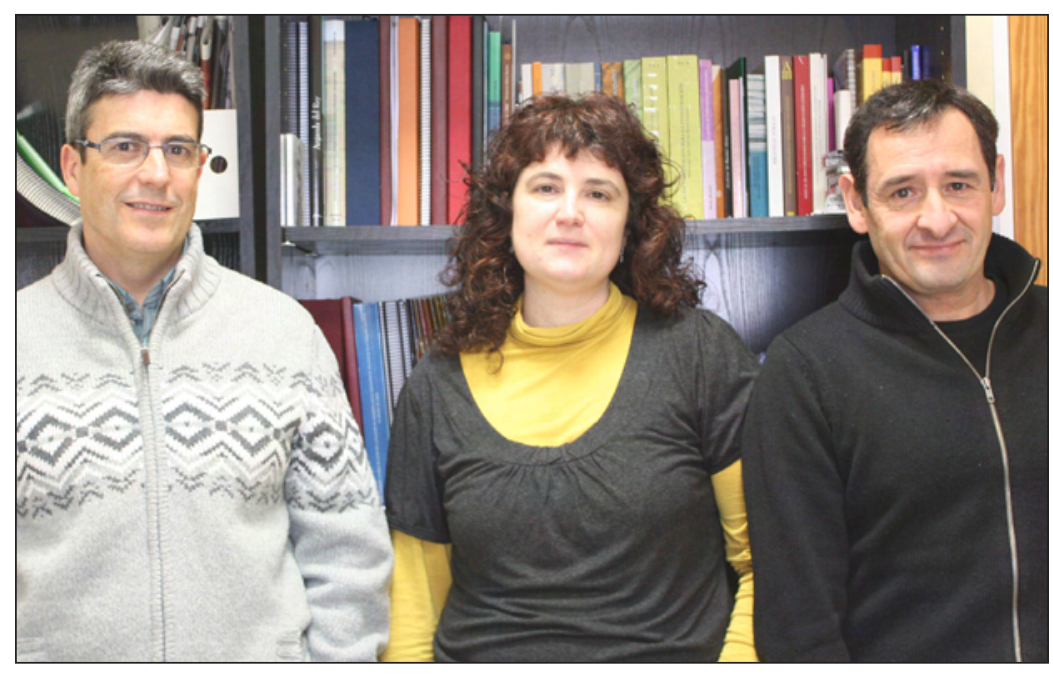

José-Antonio Cordón-García es profesor titular de la Univ. de Salamanca. Miembro del Consejo Ejecutivo del Instituto de historia del libro y de la Lectura, ha publicado numerosos artículos y monografías sobre la industria editorial en general y el libro electrónico en particular. Dirige el grupo de investigación E-Lectra: Edición y Lectura Electrónica, Transferencia y Recuperación Automatizada de la Información. Director de la revista Pliegos de Yuste: revista de ciencia, cultura y pensamiento europeos, y presidente de la Asociación Española de Bibliología. Forma parte del proyecto Territorio Ebook, de la Fundación Germán Sánchez Ruipérez.

Raquel Gómez-Díaz es profesora de la Universidad de Salamanca. Cuenta con un amplio número de publicaciones entre las que destaca la publicada junto con José-Antonio Cordón-García, Julio Alonso-Arévalo y Jesús López-Lucas Las nuevas fuentes de información. Información y búsqueda documental en el contexto de la web 2.0, así como otras relacionadas con la recuperación de la información. Asimismo ha publicado varios artículos relacionados con los libros electrónicos, e impartido cursos sobre el mismo tema. Es miembro del grupo de investigación E-Lectra y del Instituto Universitario de Estudios de la Ciencia y la Tecnología de la Universidad de Salamanca.

rgomez@usal.es

Julio Alonso-Arévalo, responsable de la Biblioteca de Traducción y Documentación de la Univ. de Salamanca y editor del repositorio E-LIS, es coordinador de la lista InfoDoc y autor de diferentes artículos en revistas especializadas sobre e-libros, OA y gestores de referencias. Ha publicado los libros: Informe APEI sobre acceso abierto, Las nuevas fuentes de información: Información y búsqueda documental en el contexto de la web 2.0, junto con José-Antonio Cordón-García, Raquel GómezDíaz y Jesús López-Lucas, y Gutenberg 2.0: la revolución de los libros electrónicos, junto con José-Antonio Cordón-García y Raquel Gómez-Díaz. Es miembro del grupo de investigación E-Lectra.

Facultad de Traducción y Documentación, Universidad de Salamanca Francisco de Vitoria, 6-16. 37008 Salamanca alar@usal.es

\section{Resumen}

La libre descarga de contenidos digitales en el entorno editorial se considera un problema por parte de los responsables culturales y empresariales de todo el mundo. La creación de sistemas de protección de archivos, la aparición de organismos nacionales e internacionales para vigilar las descargas ilegales, y la reticencia del sector editorial a dejar el papel a favor del formato digital conforman el contexto en el que tiene lugar un práctica aún poco estudiada, cuando de libros se trata. Se analizan las condiciones en que se produce esta descarga en España examinando la oferta legal de contenidos y la disponibilidad de obras en redes p 2 y y blogs. Se parte de la hipótesis que en el campo del libro las descargas de contenidos carecen de la relevancia y magnitud que revisten en el de la música y el cine, y en gran parte están motivadas por la carencia de una oferta legal variada y actualizada, y por las deficiencias de las plataformas de distribución y venta. Para ello se analiza la presencia de las obras más vendidas en las principales plataformas de venta y en diferentes redes de intercambio de archivos.

\section{Palabras clave}

Libros electrónicos, Redes p2p, DRM, Industria editorial, Libranda, España.

Title: eBooks: commercial availability and $\mathrm{p2p}$ networks 


\begin{abstract}
Practices for free digital content downloads in the publishing industry are referred to as a problem by cultural and business leaders worldwide. The creation of systems to protect the files, the emergence of national and international agencies to monitor illegal downloads, and the publishing industry's reluctance to abandon the paper and embrace the digital formats, provide the context for a practice that is little studied when it comes to books. The aim of this paper is to analyze the conditions under which it occurs in Spain, examining the legal provision of content, and the availability of works in p $2 p$ networks and blogs. The hypothesis is that in the book arena, the content downloads do not have the relevance and scale they possess in music or film, and that in large part they are motivated by the lack of a legal offering that is varied and up-to-date, and by deficiencies in the sales and distribution platforms. The presence of the bestselling books in major sales platforms and in different file sharing networks is analyzed.
\end{abstract}

\title{
Keywords
}

Ebooks, P2p, DRM, Publishing industry, Libranda, Spain.

Cordón-García, José-Antonio; Gómez-Díaz, Raquel; Alonso-Arévalo, Julio. “Libros electrónicos: oferta comercial y redes p2p". El profesional de la información, 2011, marzo-abril, v. 20, n. 2, pp. 149-158.

DOI: 10.3145/epi.2011.mar.04

\section{Introducción}

El aumento en el consumo de libros electrónicos ha endurecido la polémica sobre las descargas de contenidos ilegales, polémica trasladada a los medios políticos mediante la cuestionada Ley Sinde ${ }^{1}$, que ha reavivado las discusiones sobre unas prácticas que se van desplazando desde el sector audiovisual al editorial. Los editores de todo el mundo han iniciado procesos de digitalización de sus catálogos para que estén disponibles en la Red, al tiempo que adoptan iniciativas tendentes a la formación de colecciones digitales completamente nuevas.

El problema que se plantea el sector, que está en el trasfondo de muchas de las reticencias de las empresas a emprender esta vía, es el de la facilidad de copia y descarga de los contenidos de pago. En todos los estudios y análisis Ilevados a cabo en torno a este fenómeno encontramos esta preocupación. El informe realizado por el $O b$ servatorio del libro y la lectura sobre el libro electrónico (Observatorio, 2010), aparece repleto de alusiones a la propiedad intelectual y a las descargas ilegales por parte de los diversos actores de la cadena del libro. El estudio de Dosdoce (2009) sobre digitalización del libro y uso de redes sociales en el sector editorial incide igualmente en el tema.

El uso del DRM (digital rights management) o la distribución libre de contenidos son opciones que en estos momentos se barajan como alternativas en el sector editorial, reticente en muchos casos sobre las bondades del primero de ellos. En todo caso lo que éstos y otros estudios muestran (Stern, 2010; Reid, 2009, O'Leary, 2009; Schulze; Mochalski, 2009; OCDE, 2009; TERA, 2010) es la existencia de prácticas de consumo asociadas a los nuevos formatos que responden a lógicas diferenciadas según el contexto en el que las analicemos. La creación del Observatorio mundial de lucha contra la piratería en el seno de la Unesco en abril de 2010 es una muestra de la preocupación internacional sobre este particular:

http://portal.unesco.org/culture/es/ev.php-URL_ID=39055\& URL_DO=DO_TOPIC\&URL_SECTION=201.htm/
Lo mismo ocurre con el Observatorio europeo contra la falsificación y la piratería creado en el seno de la Unión Europea en 2009:

http://ec.europa.eu/internal_market/iprenforcement/obser vatory/index_en.htm

O el Observatorio de piratería y hábitos de consumos digitales creado en España en 2010.

http://www.cedro.org/Files/Observatoriopirateriayhabitos consumodigitales.pdf

Hay quien considera las descargas ilegales una forma de publicidad que favorece la visibilidad de los contenidos

Las descargas ilegales constituyen un fenómeno evidente y constatable en todos los sectores de las industrias culturales, percibido de manera muy distinta por los responsables de la cadena de producción de contenidos. Algunos consideran que son una forma de publicidad que favorece la visibilidad de los mismos ${ }^{2}$ y que, en todo caso, no impide que los lectores interesados dejen de acudir a los sitios web de editoriales y librerías en línea para adquirir una versión autorizada del libro. Se observa una complementariedad más que una destrucción de ventas, explicable por el hecho de que los que descargan contenidos son al mismo tiempo grandes lectores y compradores (Van-Eijk; Poort; Rutten, 2010). Los cálculos efectuados sobre la influencia de las descargas en el mercado del libro impreso suelen partir de una hipótesis falsa: que la descarga de un libro digital en una red $\mathrm{p} 2 \mathrm{p}$ (peer-to-peer, de usuario a usuario) se traduce en una compra perdida. La industria del libro tiene su referente en el sector musical en cuanto a sus reticencias por las descargas ilegales. Algunos trabajos sobre los efectos de las descargas por internet y el uso del p2p en las ventas de música, como los de Peitz y Waelbroeck (2004), Oberholzer y Strumpf (2007), TNO, SEO, IViR (2009), Rob y Waldfogel (2006) o el de Van-Eijk, Poort y Rutten (2010), constituyen una muestra de la prudencia con la que hay que acoger estas manifestaciones. 
En cuanto a los libros electrónicos se están empezando a hacer estudios, como el de Ed McCoyd (2010), que en una comunicación presentada en el encuentro sobre libros electrónicos celebrado en mayo de 2010 en el International Digital Publishers Forum (IDPF) afirma que no existe una correlación entre la descarga ilegal de libros y el descenso de ventas en el sector. Según este autor en el año 2009 se efectuaron 9 millones de descargas ilegales que afectaron a cerca de 1.000 títulos, los más populares o más vendidos entre los libros impresos.

Por su parte Attributor (2010), en un trabajo de enero de 2010 estimaba que hubo 9 millones de descargas ilegales de libros con derechos de autor en el cuarto trimestre de 2009; Attributor, que trabaja para editoriales como Hachette Book Group y John Wiley \& Sons, monitoreó 25 sitios web que ofrecen a los lectores contenidos descargables en busca de 913 títulos de sus clientes en categorías que van desde libros de negocios e inversiones a libros de ficción. Los resultados arrojaron datos como que se descargaron 1.082 copias ilegales del libro Freakonomics, de Steven D. Levitt y Stephen J. Dubner, y 7.951 de Ángeles y demonios, de Dan Brown. Se estimaba que la pérdida potencial de la industria editorial podría estar en torno a los 3.000 millones de dólares. En octubre de 2010 se llevó a cabo una segunda parte de este estudio según el cual se han incrementado en un $50 \%$ las búsquedas online para descargas ilegales, de tal manera que a diario se realizan entre 1,5 y 3 millones de consultas en Google para la búsqueda de ebooks piratas. Por otra parte se muestra un incremento del $20 \%$ en la demanda de este tipo de obras desde que el iPad salió al mercado.

En España tenemos los estudios presentados en mayo y noviembre de 2010 por el Observatorio de piratería y hábitos de consumo de contenidos digitales, impulsado por la Coalición de creadores e industrias de contenidos, una red en la que está integrada Cedro. El Observatorio encargó un estudio a la consultora independiente norteamericana IDC sobre la piratería on line en España, estudio que se pretende plantear con un carácter semestral. Según el primero, de mayo de 2010, la piratería de contenidos digitales fue superior a los 5.000 millones de euros, una cifra que representa más de un $75 \%$ del mercado de películas, videojuegos, música y libros. Respecto a éstos, se estima que un $19,7 \%$ de los contenidos puestos en la Red han sido pirateados, lo que en términos económicos representa unos 200,5 millones de euros. Una cuestión importante que recoge el estudio es que el $50 \%$ de los usuarios estarían dispuestos a pagar por la descarga de contenidos en internet. El segundo informe, de noviembre de 2010, reconoce que los libros son los contenidos menos afectados por las descargas ilegales, aunque éstas se han duplicado respecto a mayo del mismo año, alcanzando un monto económico de 421,5 millones de euros. Se aprecia también una disminución de la disposición a pagar por la descarga de contenidos digitales, un 59,4 frente al $69 \%$ del informe de mayo, lo que el sector cifra en unas pérdidas potenciales de 250 millones de euros (Ministerio de Cultura, 2010).

La cuestión es que los datos que aporta el estudio se corresponden mal con las cifras del Comercio interior del libro (Federación, 2010), la facturación de ventas de libros electrónicos alcanzó 51.259.000 euros para el año 2009, último de los informes publicados por la Federación de gremios de editores. Según la encuesta Hábitos de lectura y compra de libros para el año 2010 (Federación, 2011), del $60,3 \%$ de la población que lee libros, un 5,3\% lo hace en soporte digital, mayoritariamente pertenecientes al sector ubicado entre los 14 y los 24 años. Los compradores de libros en internet, según el mismo estudio, ascienden al $2,1 \%$, del $23 \%$ de compradores de libros no de texto para el año 2010. ¿De dónde salen los 400 millones de euros de pérdidas potenciales por las descargas ilegales? Si las cifras de facturación del libro no de texto se estiman según el mismo informe en unos 1.000 millones de euros ¿̇ignificaría esto que de no existir descargas la mitad de la población lectora se dedicaría a la compra de obras digitales? O las estadísticas de lectura están mal hechas o el análisis mal confeccionado, pero las cifras son contradictorias. Se desconoce la metodología con la que se ha llevado a cabo el estudio anterior, imprescindible para conocer sobre qué modelos se han efectuado los cálculos. Por otra parte es muy difícil realizar cuantificaciones razonables cuando se opera con universos de referencia completamente diferentes, como son los del libro en papel y el libro electrónico. Cuando la industria discográfica o la cinematográfica efectúan ponderaciones sobre pérdidas previsibles trabajan con soportes similares cuya copia puede interferir el funcionamiento del mercado. En el caso del libro electrónico se atribuyen decisiones de descarga digitales a inhibiciones de compra de formato papel, equiparando productos y procesos completamente diferenciados. Un informe de Forrester de noviembre de 2010 (McQuivey, 2010) informa que un $50 \%$ de los encuestados accede a sus lecturas mediante el préstamo interpersonal. Un 38\% afirma que encuentra sus lecturas en la biblioteca pública, y sólo un 28\% compra sus libros en Amazon. ¿Realmente creen los responsables del estudio y los editores españoles que los datos de descarga se traducirían en compras en el caso de no existir éstas? El problema en el caso de los libros (no así en el de las películas o la música), como se intentará poner de manifiesto en el artículo, es la inexistencia de un modelo de negocio adaptado al formato digital y la falta de unas ofertas suficientes, equilibradas y atractivas.

El problema, en el caso de los libros, es la inexistencia de un modelo de negocio adaptado al formato digital

Los objetivos de este artículo son:

- comprobar la presencia y naturaleza de las obras más vendidas en las plataformas de venta de libros electrónicos con objeto de comprobar su disponibilidad y las posibilidades de acceso para el lector-comprador interesado;

- comprobar su presencia en redes p2p y blogs de enlaces;

- analizar los temas y antigüedad de las obras presentes en las redes $\mathrm{p} 2 \mathrm{p}$

- analizar la representatividad de la oferta editorial de las plataformas de venta de libros electrónicos, así como su operatividad. 
Las hipótesis de partida son, por una parte, que un número importante de descargas están motivadas por una oferta insuficiente o nula de obras de actualidad que puedan suscitar el interés de los compradores potenciales. Por otra, que la incorporación de libros a las redes p2p es fundamentalmente de bestsellers, no afectando significativamente al resto de obras literarias, excepto en el campo de la literatura juvenil -en el que la literatura fantástica, la ciencia ficción y el terror tienen un presencia considerable-. Ese sesgo es imputable a la naturaleza de las personas que efectúan la digitalización de las obras, que tienen el ánimo de compartir la literatura que les gusta. Suelen ser grupos de edad de 2530 años. Esta es la razón de que en numerosas ocasiones se encuentren textos no publicados en España, directamente traducidos por redes de amigos que cuelgan versiones muy deficientes de los mismos ${ }^{3}$. Esto en el ámbito literario, en el académico o profesional la incidencia es nula.

\section{Metodología}

Se seleccionaron las obras más vendidas según el Barómetro de hábitos de lectura y compra de libros 2010 (primer cuatrimestre), esto es: El símbolo perdido, de Dan Brown ; El tiempo entre costuras, de María Dueñas; Los hombres que no amaban a las mujeres, de Stieg Larsson; Perdona, pero quiero casarme contigo, de Federico Moccia; Venganza en Sevilla, de Matilde Asensi; La reina en el palacio de las corrientes de aire y La chica que soñaba con una cerilla y un bidón de gasolina, de Stieg Larsson; La mano de Fátima, de Ildefonso Falcones; Sangre derramada, de Asa Larsson; EI secreto, de Rhonga Byrne; Contra el viento, de Ángeles Caso; El asedio, de Arturo Pérez-Reverte; La hora de los sensatos, de Leopoldo Abadía; Dime quién soy, de Julia Navarro; Lo que esconde tu nombre, de Clara Sánchez; Nueva gramática española, de la RAE; Memorias de un preso, de Mario Conde; La noche de los tiempos, de Antonio Muñoz-Molina; La isla bajo el mar, de Isabel Allende; y El niño con el pijama de rayas, de John Boyne.

Para ver la disponibilidad de los libros se eligieron dos canales de distribución. Por un lado, los que representan la vía legal: plataformas y librerías online, y por otro, lugares de intercambio de archivos como redes $p 2 p$ y otros que informan de sitios donde descargar la información.

Dentro de las plataformas y librerías se analizaron: Todoebook, Leer-e, Leqtor, y Laie en los sitios nacionales. La selección ha seguido un criterio cuantitativo: eran los sitios que ofrecían un mayor catálogo de libros electrónicos. En cuanto a las redes $p 2 p$ y blogs se tuvieron en cuenta sistemas de descarga directa en sitios de alojamiento de archivos como Megaupload, Rapidshare, 4Shared, Gigasize, etc., o sistemas intermedios como Pando. Dentro de esta categoría se encuentran: Vagos, Desde un lugar mejor o Tripilandia. En lo que se refiere a webs que informan acerca de sitios de descarga se tuvieron en cuenta los blogs Ángeles de Charlie, Mr Willian, y El último libro.

http://www.todoebook.com

http://tienda.leer-e.es

http://www.leqtor.com/es

Para este trabajo se han efectuado las búsquedas en tres momentos con objeto de medir la tasa de incorporación de ebooks a las plataformas y redes. La primera tuvo lugar en los meses de mayo-junio de 2010, la segunda en septiembre-octubre de 2010 y la tercera en diciembre de 2010. En la segunda revisión se incorporaron las obras más leídas según el Barómetro de hábitos de compra y lectura de libros correspondientes al segundo cuatrimestre de 2010 que no figuraban en la relación del primer cuatrimestre, a saber:

Crimen en directo, de Camila Lackbert; Los ojos amarillos de los cocodrilos, de Katherine Pancol; La segunda vida de Bree Tanner, de Stephene Meyer; No consigo adelgazar, de Pierre Dukan; Conspiración Octopus, de Daniel Stulin; La biblioteca de los muertos, de Glenn Cooper; El palestino, de Antonio Salas; Sé lo que estás pensando, de John Verdon; La inutilidad del sufrimiento, de María-Jesús Álava; y Ponte en forma en 9 semanas y media, de Juan Rayo.

En la tercera revisión la consulta se ha centrado únicamente en Libranda, no disponible o con escasos títulos todavía en las dos revisiones anteriores, pues en ella están incorporados los sitios que habíamos contemplado en los análisis de junio y octubre. En esta última revisión se ha tomado como referencia la relación de las mejores obras publicadas en 2010, según las estimaciones de Babelia, El cultural y otros suplementos literarios, con objeto de comprobar si el factor de arrastre atribuible a estas noticias se contempla en esta plataforma en modo de oferta editorial.

Las variables estudiadas fueron:

- existencia o no del libro en el sitio;

- fecha de publicación del libro en papel;

- fecha de publicación del post en los sitios p2p;

- fecha de publicación del libro en las tiendas online;

- precio del libro en papel;

- precio del libro online;

- formatos de los ficheros;

- existencia o no de DRM en las tiendas online;

- tipos de DRM (si admite o no la copia, si admite o no la impresión, si admite o no el préstamo).

La tasa de incorporación de obras a las redes y las características de estas obras constituyen una información esencial para apreciar los componentes estructurales de la práctica de la descarga. Para analizar estos supuestos se examinaron las obras incorporadas a 4 sitios y blogs de descargas durante los meses de septiembre, octubre y diciembre de 2010 , comprobando los títulos, su antigüedad y las editoriales afectadas. La hipótesis de partida es que en la mayoría de los casos se trata de obras con varios años de antigüedad cuya disponibilidad en la Red afecta mínimamente al mercado editorial al ser obras cuyo ciclo vital está en fase declinante. Se analizaron las 100 primeras obras que figuran en los blogs y redes Ángeles de Charlie, El Último Libro, Mr Willian y Vagos, debido a que son las que cuentan con un mayor número de descargas. Por otra parte se comprobó la presencia de estas obras en los sitios legales para analizar el grado de competencia que puede existir a igualdad de formatos.

Con el fin de comprobar las distribuciones de frecuencias y la correlación existente entre todas las variables, los datos se trataron con el programa estadístico Statview. 


\section{Resultados}

\section{Primera revisión}

Los resultados obtenidos fueron significativos ya que ninguna de las obras seleccionadas se encontraba presente en Todoebook, ni Leqtor ni Leer-e. Sólo Laie tenía las obras de Julia Navarro, Ildefonso Falcones y María Dueñas.

Por el contrario la presencia de las obras es frecuente en todas las redes $\mathrm{p} 2 \mathrm{p}$ analizadas, como se puede apreciar en la tabla 1.

\begin{tabular}{|l|c|l|c|}
\hline \multicolumn{1}{|c|}{ OBRAS } & $\begin{array}{c}\mathrm{n}^{\circ} \text { de } \\
\text { redes } \\
\text { p2p }\end{array}$ & \multicolumn{1}{|c|}{ OBRAS } & $\begin{array}{c}\mathbf{n}^{\circ} \text { de } \\
\text { redes } \\
\text { p2p }\end{array}$ \\
\hline Contra el viento & 5 & La mano de Fátima & 7 \\
\hline Dime quién soy & 4 & La noche de los tiempos & 3 \\
\hline El asedio & 6 & $\begin{array}{l}\text { La reina en el palacio de } \\
\text { las corrientes... }\end{array}$ & 5 \\
\hline $\begin{array}{l}\text { El niño con el pijama } \\
\text { de rayas }\end{array}$ & 5 & $\begin{array}{l}\text { Lo que esconde tu } \\
\text { nombre }\end{array}$ & 4 \\
\hline El secreto & 6 & $\begin{array}{l}\text { Los hombres que no } \\
\text { amaban a las mujeres }\end{array}$ & 6 \\
\hline El símbolo perdido & 7 & Memoria de un preso & 0 \\
\hline El tiempo entre costuras & 4 & $\begin{array}{l}\text { Nueva gramática de la } \\
\text { lengua española }\end{array}$ & 0 \\
\hline $\begin{array}{l}\text { La chica que soñaba } \\
\text { con una cerilla... }\end{array}$ & 6 & $\begin{array}{l}\text { Perdona, pero quiero } \\
\text { casarme contigo }\end{array}$ & 3 \\
\hline La hora de los sensatos & 2 & Sangre derramada & 4 \\
\hline La isla bajo el mar & 4 & Venganza en Sevilla & 5 \\
\hline
\end{tabular}

Tabla 1. Disponibilidad de 20 títulos en 7 redes p2p (mayo-junio de 2010)

Sólo dos obras, Memorias de un preso, de Mario Conde, y la Nueva gramática de la lengua española no figuran en ninguna de las redes analizadas. El número de descargas medias por título está entre las 600 y las 2.000 , aunque este dato es relativo dado que no todas las redes lo incluyen.

Los formatos de archivo más frecuentes son el pdf y el doc, así como la combinación pdf, fb2 y pdf, doc.

El paso de un título publicado en papel a una red p2p tarda entre 1 y 3 años en el 90\% de los casos, excepto los bestsellers cuya incorporación es inmediata.

\section{Segunda revisión}

Se observaron cambios en las plataformas y tiendas online, que pasaron de una oferta de los títulos seleccionados prácticamente inexistente a una presencia mucho más importante. Todoebook por ejemplo, ha incorporado Los hombres que no amaban a las mujeres, La mano de Fátima, Dime quién soy, Memorias de un preso y La noche de los tiempos, que no figuraban en la primera consulta. En conjunto han pasado de poseer sólo tres títulos del conjunto de 20 que se tuvieron en cuenta en la primera fase a tener la mitad de los 30 que se han considerado en la segunda fase.

Laie disponía de 12 títulos, Lector 12, Todoebook 8 y únicamente en Leer-e no se encontró ninguno (tabla 2 -columna 2-).

\begin{tabular}{|c|c|c|}
\hline OBRAS & $\begin{array}{c}\mathrm{n}^{\circ} \mathrm{de} \\
\text { tiendas }\end{array}$ & $\begin{array}{c}\mathrm{n}^{\circ} \mathrm{de} \\
\text { redes } \\
\mathrm{p} 2 \mathrm{p}\end{array}$ \\
\hline Conspiración Octopus & 2 & 1 \\
\hline Contra el viento & 0 & 5 \\
\hline Crimen en directo & 0 & 4 \\
\hline Dime quién soy & 3 & 4 \\
\hline El asedio & 0 & 5 \\
\hline El niño con el pijama de rayas & 0 & 5 \\
\hline Elpalestino & 0 & 0 \\
\hline El secreto & 1 & 5 \\
\hline El símbolo perdido & 0 & 6 \\
\hline El tiempo entre costuras & 2 & 4 \\
\hline La biblioteca de los muertos & 3 & 4 \\
\hline La chica que soñaba con una cerilla... & 2 & 6 \\
\hline La hora de los sensatos & 2 & 2 \\
\hline La inutilidad del sufrimiento & 0 & 0 \\
\hline La isla bajo el mar & 0 & 4 \\
\hline La mano de Fátima & 3 & 6 \\
\hline La noche de los tiempos & 3 & 4 \\
\hline La reina en el palacio de las corrientes... & 2 & 5 \\
\hline La segunda vida de Bree Tanner & 0 & 4 \\
\hline Lo que esconde tu nombre & 0 & 4 \\
\hline Los hombres que no amaban a las mujeres & 3 & 6 \\
\hline Los ojos amarillos de los cocodrilos & 0 & 3 \\
\hline Memoria de un preso & 3 & 0 \\
\hline No consigo adelgazar & 0 & 1 \\
\hline Nueva gramática de la lengua española & 0 & 0 \\
\hline Perdona, pero quiero casarme contigo & 0 & 3 \\
\hline Ponte en forma en nueve semanas y media & 0 & 0 \\
\hline Sangre derramada & 0 & 4 \\
\hline Sé lo que estás pensando & 3 & 3 \\
\hline Venganza en Sevilla & 0 & 5 \\
\hline
\end{tabular}

Tabla 2. Disponibilidad de los 30 títulos en las tiendas online y en redes $p 2 p$ (sept.-octubre de 2010)

Todos los archivos están protegidos con $D R M$ y se impide la copia y la impresión. El formato más frecuente es epub.

Los precios de los libros son idénticos en todos los sitios consultados, por debajo de los precios en papel en todos los casos, pero todavía muy lejos de los precios que los lectores están dispuestos a pagar por un archivo digital (tabla 3).

En las consultas efectuadas a Amazon e Ibookstore no se ha encontrado ninguno de los títulos en español disponible para descarga en dispositivos de lectura, aunque sí en papel. En justa proporción, el iPad y el Kindle no son compati-

\begin{tabular}{|l|c|c|c|c|c|c|}
\hline Soporte & $\begin{array}{c}\text { Media } \\
(€)\end{array}$ & $\begin{array}{c}\text { Des- } \\
\text { viación } \\
\text { típica }\end{array}$ & $\begin{array}{c}\text { Desvia- } \\
\text { ción del } \\
\text { error }\end{array}$ & $\begin{array}{c}\text { Número } \\
\text { de datos } \\
\text { tenidos en } \\
\text { cuenta }\end{array}$ & $\begin{array}{c}\text { Precio } \\
\text { mínimo } \\
(€)\end{array}$ & $\begin{array}{c}\text { Precio } \\
\text { máximo } \\
(€)\end{array}$ \\
\hline Papel & 20,34 & 3,252 & 0,349 & 87 & 12,02 & 27,88 \\
\hline Digital & 14,42 & 2,165 & 0,377 & 33 & 10,99 & 17,49 \\
\hline
\end{tabular}

Tabla 3. Comparación de precios papel/digital 
bles en ninguna de las tiendas españolas para descargas de obras digitalizadas.

Respecto a las redes p2p se observó en esta segunda fase del estudio un comportamiento diferente en la subida de títulos. Las incorporaciones son menos numerosas en todas las redes, excepto en una generalista, Vagos y otra especializada, Ángeles de Charlie. El resto, o bien no ha subido ninguna de las obras pertenecientes a la segunda encuesta de 2010 sobre hábitos de lectura (caso de Mr William) o lo han hecho muy tímidamente, como ocurre con Desde un lugar mejor. El número de descargas también ha disminuido casi a la mitad. En algunos casos se observa una política de prudencia en la incorporación de nuevos títulos, optando en su mayoría por obras que poseen un recorrido editorial de más de un año, como si se estuviera aplicando un embargo implícito a las obras digitalizadas. De todos modos la proporción en cuanto a presencia es mayor que en las tiendas analizadas antes (tabla 2 -columna 3-, y tabla 4).

Los formatos de archivo más frecuentes son epub, pdf, doc y fb2

\begin{tabular}{|l|c|}
\hline Vagos & 23 \\
\hline El último libro & 21 \\
\hline Taringa & 21 \\
\hline Ángeles de Charlie & 20 \\
\hline Desde un lugar & 9 \\
\hline Tripilandia & 9 \\
\hline
\end{tabular}

Tabla 4. Número de obras en 6 redes $p 2 p$

La distribución temporal de las 100 obras analizadas en los cuatro sitios seleccionados nos indica que se reparten en un período de tiempo prolongado, una década aproximadamente (gráfico 1).

Las obras se distribuyen también entre varias decenas de editoriales sin que destaque ninguna (tabla 5). Únicamente Alfaguara, editorial de Mario Vargas Llosa, ha disfrutado de múltiples subidas tras ser galardonado este escritor con el Premio Nobel. En las tiendas de venta de ebooks se han encontrado solamente 6 de las 100 obras.

\section{Tercera revisión}

Tuvo lugar en diciembre de 2010 y no aportó cambios significativos respecto a lo observado en las anteriores. Dado que en este mes se publicaron varios informes sobre los me-

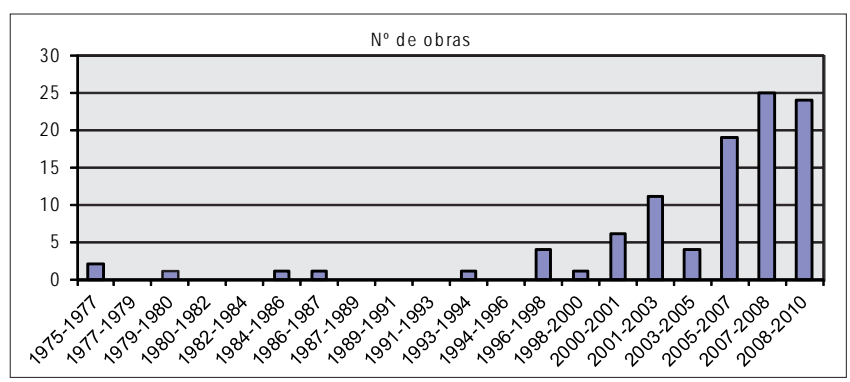

Gráfico 1. Distribución de ebooks por año de publicación del libro

\begin{tabular}{|c|c|c|c|}
\hline Editorial & $n^{\circ}$ ebooks & Editorial & $n^{\circ}$ ebooks \\
\hline Alfaguara & 12 & Minotauro & 1 \\
\hline Alianza & 1 & Mondadori & 3 \\
\hline Anagrama & 2 & Nebulae & 1 \\
\hline Arlequín & 1 & Península & 1 \\
\hline Astri & 1 & Phoebe & 1 \\
\hline Belacqua & 1 & Planeta & 4 \\
\hline Bibliópolis & 2 & Plaza y Janés & 5 \\
\hline$B S$ & 1 & Prentice Hall & 1 \\
\hline Círculo de Lectores & 1 & Punto de Lectura & 2 \\
\hline Continente & 1 & Puzzle & 1 \\
\hline Debolsillo & 5 & $R B A$ & 3 \\
\hline Edhasa & 1 & Roca & 2 \\
\hline Ediciones $B$ & 7 & Roca Bolsillo & 1 \\
\hline El Acantilado & 1 & Salamandra & 4 \\
\hline El Aleph & 1 & Seix Barral & 1 \\
\hline Emece & 1 & Siruela & 2 \\
\hline Espasa & 1 & Suma & 3 \\
\hline Factoría & 3 & Temas de hoy & 1 \\
\hline Factoría Ideas & 1 & Terciopelo & 1 \\
\hline Grijalbo & 4 & Timun Mas & 1 \\
\hline Hiria & 1 & Tusquets & 4 \\
\hline Intercontinental & 1 & Umbriel & 1 \\
\hline Lumen & 1 & Valdemar & 1 \\
\hline Maeva & 1 & Viamagna & 1 \\
\hline Martínez Roca & 1 & Zeta & 1 \\
\hline
\end{tabular}

Tabla 5. Disponibilidad de los 100 primeros títulos en redes p2p según editorial

\section{La oferta en las plataformas de venta y distribución de libros electrónicos es es- casa y poco actualizada}

jores libros del año en opinión de críticos y revistas especializadas, se analizó la presencia de estas obras en Libranda, el portal español de venta de libros electrónicos propiciado por los principales grupos editoriales del país (Santillana, Planeta, Mondadori, etc.) que incluía, además, los sitios web anteriormente analizados (Todoebook, Laie, Leqtor, Leer-e). Se pretendía comprobar si, como ocurre con el factor de arrastre que representan esos informes en el caso del libro en papel (López-De-Abiada; Peñate-Rivero, 1997; López-De-Abiada; Neuschafer; López-Bernasocchi, 2001), las editoriales probarían con el libro digital, teniendo en cuenta que la mayoría de lo libros pertenecen a editoriales de los grupos implicados en Libranda, como Alfaguara, Mondadori, Anagrama, Taurus, Tusquets, Siruela, etc. Se comprobó igualmente la presencia de estas obras en Amazon y en Barnes \& Noble en español, portal de venta de libros electrónicos en nuestro idioma lanzado en noviembre de 2010. Dado que una de las características de la edición electrónica es la ruptura del modelo de territorialidad sobre el que se asienta la edición en papel, era necesario analizar si la ausencia de obras en español en otros portales podía representar un re- 
ferente de competencia para los compradores potenciales. Se comprobaron igualmente sistemas de $D R M$ y precios de los libros.

La consulta a Libranda confirma las irregularidades ya señaladas por algunos autores ${ }^{4,5}$. . El sistema es complejo. Cuando se busca una obra, la plataforma remite a las librerías que presumiblemente la tienen y en casi la mitad de ellas, como se puede apreciar en la tabla 6, no aparece la obra sino el catálogo de la librería, por lo que hay que volver a efectuar la búsqueda. El sistema de información de las tiendas es igualmente deficiente. En muchas de ellas no se informa sobre el formato de las obras o sobre el $D R M$ y en casi ninguna sobre los dispositivos compatibles.

\section{Las obras más vendidas en papel tienen poca presencia en formato digital, favo- reciendo el recurso a las redes $\mathrm{p} 2 \mathrm{p}$}

Respecto a la consulta de los títulos más valorados en 2010, los resultados son significativos. No hay ni uno de ellos en Libranda, ni el título buscado ni ninguna obra de ese autor. Esto es especialmente grave en el caso de Vargas Llosa o Herta Müller, ambos premios Nobel, cuyos libros en formato digital sí que se pueden encontrar en Amazon o en Barnes \& Noble en español. Sin embargo todos los títulos excepto dos se pueden encontrar en alguna de las redes $\mathrm{p} 2 \mathrm{p}$ o los blogs de libros mencionados anteriormente.

La muestra evidencia que, a diferencia de Libranda, un lector puede encontrar libros electrónicos de los autores que se han tomado como referencia en Barnes \& Noble y en Amazon. Estas librerías ofrecen además otros títulos de los autores (tabla 7).

Como ya se ha indicado, esos títulos se encuentran en alguna de las redes $\mathrm{p} 2 \mathrm{p}$ y blogs especializados que se han consultado, excepto el de Todorov y el de Aksiónov.

\section{Conclusiones}

La oferta en las plataformas de venta y distribución de libros electrónicos es escasa y poco actualizada. Las obras más vendidas en papel tienen poca presencia en formato digital, favoreciendo el recurso a las redes p2p por parte de aquellos interesados en la lectura digital.

Las plataformas de venta, especialmente Libranda, cuentan con un catálogo reducido (en comparación con los títulos en papel de los grupos y editoriales que la alimentan), poco representativo y de compleja usabilidad. La reproducción de un sistema tradicional de distribución en un contexto digital, como es el caso de Libranda, dificulta la consulta, el acceso a las obras y perjudica la visibilidad de las librerías asociadas por cuanto las complicaciones del proceso pueden provocar un efecto de rechazo en los clientes potenciales, generando la búsqueda alternativa en otros sitios.

Todas las obras presentes en las plataformas de venta cuentan con sistemas de protección DRM que suponen una primera barrera contra la piratería. Frente a las posibilidades de uso del archivo impuestas por el distribuidor, el lector puede sentir la tentación de recurrir a ofertas carentes de estas limitaciones, operando con el libro electrónico en las mismas circunstancias que lo haría con un ejemplar en papel.

\begin{tabular}{|c|c|c|c|c|c|c|}
\hline Abacus & Bertrand & $\begin{array}{l}\text { Proteo y } \\
\text { Prometeo }\end{array}$ & Bubok & El Corte Inglés & $\begin{array}{c}\text { Hijos de Santiago } \\
\text { Rodríguez }\end{array}$ & La central \\
\hline $\begin{array}{l}\text { Salta el título. No } \\
\text { informa sobre } \\
\text { formato ni sobre } \\
\text { DRM. }\end{array}$ & Salta el catálogo. & $\begin{array}{l}\text { Salta el título. } \\
\text { Formato epub. } \\
\text { DRM adobe. }\end{array}$ & $\begin{array}{l}\text { Salta el título. } \\
\text { No informa sobre } \\
\text { formato, ni sobre } \\
\text { DRM. }\end{array}$ & $\begin{array}{l}\text { Salta el título. } \\
\text { epub con Adobe } \\
\text { DRM. No permite } \\
\text { la impresión ni la } \\
\text { copia. }\end{array}$ & $\begin{array}{l}\text { Salta el título. } \\
\text { epub, no permite } \\
\text { copiar ni pegar. }\end{array}$ & $\begin{array}{l}\text { Sale el catálogo } \\
\text { de novedades, } \\
\text { pero no el título } \\
\text { buscado. }\end{array}$ \\
\hline Popularebook & Fnac & Luarna & Edicat & Leqtor & Leer-e & Laie \\
\hline $\begin{array}{l}\text { Salta el libro. In- } \\
\text { forma de formato } \\
\text { y dispositivos } \\
\text { compatibles, } \\
\text { pero no de DRM. }\end{array}$ & $\begin{array}{l}\text { Salta el libro. } \\
\text { Informa de } \\
\text { formato pero no } \\
\text { de sistemas de } \\
\text { DRM. }\end{array}$ & $\begin{array}{l}\text { Salta el libro. In- } \\
\text { forma de formato } \\
\text { y } D R M \text {. }\end{array}$ & $\begin{array}{l}\text { Salta el catálogo. } \\
\text { No el título } \\
\text { buscado. }\end{array}$ & $\begin{array}{l}\text { Salta el libro. Pero } \\
\text { no informa de } \\
\text { DRM. }\end{array}$ & Salta el catálogo. & $\begin{array}{l}\text { Salta el libro. } \\
\text { Informa de for- } \\
\text { mato y } D R M \text {. No } \\
\text { permite copiar ni } \\
\text { pegar. }\end{array}$ \\
\hline Abac & Todoebook & Santos y Ochoa & Grammata & Cervantes & Diógenes & Cámara \\
\hline Salta el catálogo. & $\begin{array}{l}\text { Salta el libro. In- } \\
\text { forma de formato } \\
\text { y } D R M \text {. }\end{array}$ & $\begin{array}{l}\text { Informa de que el } \\
\text { libro no está en } \\
\text { su base de datos. }\end{array}$ & $\begin{array}{l}\text { Salta el título. } \\
\text { No informa de } \\
\text { formato ni de } \\
\text { DRM. }\end{array}$ & $\begin{array}{l}\text { Salta el catálogo } \\
\text { de novedades. }\end{array}$ & $\begin{array}{l}\text { Salta el catálogo } \\
\text { de novedades. }\end{array}$ & $\begin{array}{l}\text { Salta el catálogo } \\
\text { de novedades. }\end{array}$ \\
\hline $\begin{array}{c}\text { Antonio } \\
\text { Machado }\end{array}$ & Casa del Libro & 80 Mundos & & & & \\
\hline $\begin{array}{l}\text { Salta el libro. Pero } \\
\text { no informa de } \\
\text { formato. Prohíbe } \\
\text { copiar y pegar. }\end{array}$ & $\begin{array}{l}\text { Salta el libro. } \\
\text { Informa de } \\
\text { formato pero no } \\
\text { de } D R M \text {. Informa } \\
\text { de dispositivos } \\
\text { compatibles. }\end{array}$ & Salta el catálogo. & & & & \\
\hline
\end{tabular}

Tabla 6. Librerías a las que remite Libranda con indicación del resultado (diciembre 2010) 


\begin{tabular}{|c|c|c|c|c|c|c|}
\hline & Libranda & $\begin{array}{l}\text { Otras obras del } \\
\text { autor en Libranda }\end{array}$ & Amazon & $\begin{array}{l}\text { Otras obras del } \\
\text { autor en Amazon }\end{array}$ & $\begin{array}{l}\text { Barnes \& } \\
\text { Noble }\end{array}$ & $\begin{array}{l}\text { Otras obras del autor } \\
\text { en Barnes \& Noble }\end{array}$ \\
\hline $\begin{array}{l}\text { Verano, de J. M. Coetzee. } \\
\text { Mondadori }\end{array}$ & No & $\begin{array}{l}\text { Ninguna, en ningún } \\
\text { formato }\end{array}$ & Sí, en inglés & 2 títulos para Kindle & No & $\begin{array}{l}5 \text { títulos para NookBo- } \\
\text { ok, en inglés }\end{array}$ \\
\hline $\begin{array}{l}\text { Poesías reunidas, de Yeats. } \\
\text { Pre-Textos }\end{array}$ & No & $\begin{array}{l}\text { Ninguna, en ningún } \\
\text { formato }\end{array}$ & Sí, en inglés & $\begin{array}{l}16 \text { títulos para } \\
\text { Kindle }\end{array}$ & No & $\begin{array}{l}28 \text { títulos para Nook- } \\
\text { Book, en inglés }\end{array}$ \\
\hline $\begin{array}{l}\text { Blanco nocturno, de Ricardo } \\
\text { Piglia. Anagrama }\end{array}$ & No & $\begin{array}{l}\text { Ninguna, en ningún } \\
\text { formato }\end{array}$ & $\begin{array}{l}\text { Sí, en } \\
\text { castellano }\end{array}$ & No & $\begin{array}{l}\text { Sí, en } \\
\text { castellano }\end{array}$ & $\begin{array}{l}\text { El último lector, en cas- } \\
\text { tellano, para NookBook }\end{array}$ \\
\hline $\begin{array}{l}\text { El sueño del celta, de Mario } \\
\text { Vargas Llosa. Alfaguara }\end{array}$ & No & $\begin{array}{l}\text { Ninguna, en ningún } \\
\text { formato }\end{array}$ & No & $\begin{array}{l}\text { Los jefes y los cacho- } \\
\text { rros en castellano, } \\
\text { y La tentación de lo } \\
\text { imposible en inglés, } \\
\text { para Kindle. }\end{array}$ & No & $\begin{array}{l}\text { Los jefes y Los cachorros } \\
\text { en castellano, y La } \\
\text { tentación de lo impo- } \\
\text { sible en inglés para } \\
\text { NookBook }\end{array}$ \\
\hline $\begin{array}{l}\text { El amor verdadero, de José } \\
\text { María Guelbenzu. Siruela }\end{array}$ & No & $\begin{array}{l}\text { Ninguna, en ningún } \\
\text { formato }\end{array}$ & No & No & $\begin{array}{l}\text { Sí, en } \\
\text { castellano }\end{array}$ & $\begin{array}{l}2 \text { títulos para NookBo- } \\
\text { ok, en castellano }\end{array}$ \\
\hline $\begin{array}{l}\text { Dublinesca, de Enrique Vila- } \\
\text { Matas, Anagrama } \\
\text { Premio Leteo } 2010 \text { y Premio } \\
\text { Jean Carrière } 2010 \\
\end{array}$ & No & $\begin{array}{l}\text { Ninguna, en ningún } \\
\text { formato }\end{array}$ & No & No & No & No \\
\hline $\begin{array}{l}\text { Retratos y encuentros, de } \\
\text { Gay Talese. Alfaguara }\end{array}$ & No & $\begin{array}{l}\text { Ninguna, en ningún } \\
\text { formato }\end{array}$ & Sí, en inglés & 6 títulos para Kindle & Sí, en inglés & $\begin{array}{l}6 \text { títulos para NookBo- } \\
o k \text {, en inglés }\end{array}$ \\
\hline $\begin{array}{l}\text { Algo va mal, de Tony Judt. } \\
\text { Taurus }\end{array}$ & No & $\begin{array}{l}\text { Ninguna, en ningún } \\
\text { formato }\end{array}$ & Sí, en inglés & 4 títulos para Kindle & Sí, en inglés & $\begin{array}{l}6 \text { títulos para NookBo- } \\
\text { ok, en inglés }\end{array}$ \\
\hline $\begin{array}{l}\text { Tierra desacostumbrada, de } \\
\text { Jhumpa Lahiri. Salamandra }\end{array}$ & No & $\begin{array}{l}\text { Ninguna, en ningún } \\
\text { formato }\end{array}$ & Sí, en inglés & No & Sí, en inglés & $\begin{array}{l}3 \text { títulos para NookBo- } \\
o k \text {, en inglés }\end{array}$ \\
\hline $\begin{array}{l}\text { Correr, de Jean Echenoz. } \\
\text { Anagrama. }\end{array}$ & No & $\begin{array}{l}\text { Ninguna, en ningún } \\
\text { formato }\end{array}$ & No & No & Sí, en inglés & No \\
\hline $\begin{array}{l}\text { Todo lo que tengo lo llevo } \\
\text { conmigo, de Herta Müller. } \\
\text { Siruela }\end{array}$ & No & $\begin{array}{l}\text { Ninguna, en ningún } \\
\text { formato }\end{array}$ & No & No & $\begin{array}{l}\text { Sí, en } \\
\text { castellano }\end{array}$ & $\begin{array}{l}\text { La bestia del corazón, } \\
\text { en castellano y uno en } \\
\text { inglés para NookBook }\end{array}$ \\
\hline $\begin{array}{l}\text { La experiencia totalitaria, de } \\
\text { Tzvetan Todorov. Galaxia } \\
\text { Gutenberg. }\end{array}$ & No & $\begin{array}{l}\text { Ninguna, en ningún } \\
\text { formato }\end{array}$ & Sí, en inglés & $\begin{array}{l}\text { Un título para Kin- } \\
\text { dle, en inglés }\end{array}$ & No & $\begin{array}{l}2 \text { títulos para NookBo- } \\
\text { ok, en inglés }\end{array}$ \\
\hline $\begin{array}{l}\text { Leviatan o la ballena, de Phi- } \\
\text { lip Hoare. Ático de los libros }\end{array}$ & No & $\begin{array}{l}\text { Ninguna, en ningún } \\
\text { formato }\end{array}$ & Sí, en inglés & No & Sí, en inglés & No \\
\hline $\begin{array}{l}\text { Una saga moscovita, de Va- } \\
\text { sili Aksiónov. La otra orilla. }\end{array}$ & No & $\begin{array}{l}\text { Ninguna, en ningún } \\
\text { formato }\end{array}$ & No & No & No & No \\
\hline $\begin{array}{l}\text { La idea de la justicia, de } \\
\text { Amartya Sen. Taurus }\end{array}$ & No & $\begin{array}{l}\text { Ninguna, en ningún } \\
\text { formato }\end{array}$ & Sí, en inglés & 4 títulos para Kindle & No & $\begin{array}{l}5 \text { títulos para NookBo- } \\
\text { ok, en inglés }\end{array}$ \\
\hline $\begin{array}{l}\text { La muerte del adversario, de } \\
\text { Hans Keilson. Minúscula }\end{array}$ & No & $\begin{array}{l}\text { Ninguna, en ningún } \\
\text { formato }\end{array}$ & No & No & Sí, en inglés & $\begin{array}{l}1 \text { título para NookBo- } \\
o k \text {, en inglés }\end{array}$ \\
\hline
\end{tabular}

Tabla 7. Comparativa sobre la disponibilidad de los títulos más valorados en 2010

Las obras más vendidas están presentes en las redes y blogs de enlaces, principalmente en aquellas especializadas en libros.

En la mayoría de ocasiones estas redes suben las obras literarias al cabo de dos o tres años de su aparición en papel, excepto en el caso de los bestsellers cuya incorporación es casi inmediata.

Los formatos más generalizados son epub, pdf, doc y fb2.

La edición científica carece de representatividad tanto en las plataformas de venta como en las redes $\mathrm{p} 2 \mathrm{p}$. Esto no quiere decir que este importante subsector editorial sea invulnerable a la piratería, más bien apunta a que lo mismo que existe una diversificación y fragmentación en el lado de la oferta, se produce una diversificación de circuitos desde la parte de la demanda o la recepción.

De cualquier modo, en España la práctica de descarga de libros es poco intensiva. Frente a un $50 \%$ de los internautas que realizan habitualmente descargas en internet, los que utilizan la Red para leer/descargar libros no llegan al $9 \%$.

Si esto es todo lo que la industria editorial da de sí, definitivamente es que... no se ha entendido internet (Espada, 2010)

\section{Notas}

1. La Ley Sinde es el nombre informal con el cual se conoce a un apartado particular de la Ley de Economía Sostenible llamado "Disposición final segunda", en que el Gobierno de Rodríguez-Zapatero intenta enfrentar la crisis que atraviesa España. Fue propuesta en mayo de 2009.

Dicha Disposición consiste en una serie de cambios que afectan a: 
Ley de Servicios de la Sociedad de la Información (más conocida como $L S S I$ ).

Ley de Propiedad Intelectual.

Ley Reguladora de la Jurisdicción Contencioso-Administrativa, (que se centra en la protección de la propiedad intelectual en el ámbito de la sociedad de la información y de comercio electrónico).

El objetivo de modificar estas leyes es permitir que un grupo de personas dependientes del Ministerio de Cultura (una comisión cultural o comisión de propiedad intelectual), tengan la potestad de cerrar páginas web que, según su criterio, vulneren los derechos propiedad intelectual, previa autorización de los Juzgados Centrales de lo Contencioso Administrativo

Los cambios, como es de suponerse, vienen propuestos por la Ministra de Cultura, Ángeles González-Sinde, motivo por el cual se ganó el nombre de Ley Sinde.

Fuente: http://alt1040.com/2011/01/que-es-la-ley-sinde

2. El último ganador del Premio Alfaguara de novela se vanagloriaba en una entrevista de que sus obras circularan por las redes p2p pues esto favorecía la difusión, e indirectamente, la venta de las mismas. Cory Doctorow, autor de Little brother, obra que estuvo varias semanas en la lista de libros más vendidos del New York times, conocía tres versiones electrónicas de su libro el mismo día que se publicó en papel. Su opinión es que las mismas logran captar nuevos lectores. Según este autor el problema no es la piratería "es la oscuridad" (Rich, 2009).

3. Valga como ejemplo este texto, elegido al azar, de la traducción "colegiada" no oficial del primer libro de la serie Luna llena: "Ciertamente estaba de malas ese día en especial; mañana cumpliría los dieciocho años y tenía un mundo de tarea por terminar, no solo de la escuela, también de su 'trabajo' si se le podría decir así; realmente no iba hacer un cumpleaños fabuloso ni tampoco interesante, la escuela no le era complicada pero si muy tediosa a veces, sino fuera por sus amigos que la hacían reír todos los días sería otro zombi mas sumido al yugo escolar".

4. De Libranda, la plataforma digital made in Spain que reunió en el momento de su lanzamiento la oferta digital de 16 grupos editoriales, 90 sellos y 22 tiendas, se ha dicho mucho y prácticamente nada bueno. Que sus previsiones eran exageradas; que no vende directamente los libros sino que redirecciona a la tienda de la web de la editorial que toque (lo que obliga a realizar la búsqueda dos veces); que los precios son sólo entre un 20 y un $30 \%$ inferiores a los de las ediciones de papel; que los ebooks a la venta son sólo archivos digitalizados del original de papel, sin ningún valor añadido; que su usabilidad es, como mínimo, grumosa; etcétera, etcétera y etcétera. Pero lo peor de todo es que Libranda era la gran iniciativa de la industria editorial española. Si esto es todo lo que la industria da de sí, definitivamente es que... no se ha entendido internet" (Espada, 2010).

5. “Imaginen una librería (de las de papel de toda la vida) en la que usted quisiese entrar y fuese difícil encontrar la puerta (en algunos casos prácticamente imposible); imaginen que una vez dentro resultase que hay muy pocos libros y que algunos, misteriosamente, hubiesen perdido sus portadas; imaginen también que fuese extraordinariamente difícil encontrar un volumen determinado porque las estanterías no están bien ordenadas o que, por ejemplo, cuando preguntase al tendero por los libros de Pamuk le respondiese que no puede buscar por autor [...] Procesos complejos cuando no imposibles, decisiones difícilmente justificables desde el punto de vista del usuario, políticas de precios enloquecidas y sobre todo, no me cansaré de señalar esto, un catálogo que más que escaso es raquítico, meramente simbólico y poco menos que ridículo tanto por su volumen..." (Jordá, 2010).

6. “Resulta increíble que las editoriales más grandes de nuestro país hayan creado una plataforma gigante de libros digitales cuyo objetivo parece ser el de no vender libros digitales. El problema es que lo disimulan porque usted no se da cuenta de que no quieren venderle hasta el quinto o sexto paso, o hasta la quinta o sexta librería cibernética..." (Millás, 2010).

\section{Bibliografía}

"A first look at demand for pirated ebooks across the Web". Attributor, 6 Oct 2010.

http://attributor.com/blog/a-first-look-at-demand-for-pira ted-e-books-across-the-web/

Dosdoce.com; Ediciona. "La digitalización del libro en España". Dosdoce.com, 2009.

http://www.dosdoce.com/upload/ficheros/noticias/201 005/0000000042.pdf

Espada, Arcadi. Los diez pecados editoriales, 14 nov 2010. http://www.elmundo.es/blogs/elmundo/elmundoporden tro/2010/11/14/los-10-pecados-editoriales.html

Federación de Gremios de Editores de España. Comercio interior del libro 2009. Madrid, 2010.

http://www.federacioneditores.org/0_Resources/Documen tos/Comercio_Interior_2009.pdf

Federación de Gremios de Editores de España. Hábitos de compra y lectura de libros en España, 2010. Madrid, 2011. http://www.federacioneditores.org/0_Resources/Documen tos/NP_Lectura2010_V3.pdf

Jordá, Carmelo. "Fiasco Libranda". Libertad digital, julio 2010.

http://www.libertaddigital.com/opinion/carmelo-jorda/ fiasco-libranda-55584

López-De-Abiada, José-Manuel; Peñate-Rivero, Julio. Éxitos de ventas y calidad literaria. Madrid: Verbum, 1997.

López-De-Abiada, José-Manuel; Neuschafer, Hans; LópezBernasocchi, Augusta. Entre el ocio y el negocio: industria editorial y literatura. Madrid: Verbum, 2001

McCoyd, Ed. Digital piracy of books. IDPF digital book 2010.

McQuivey, James L. "Ebook buying is about to spiral upward". Forrester Research, Nov. 2010.

http://www.forrester.com/rb/Research/ebook_buying_is_ about_to_spiral_upward/q/id/57664/t/2

Millás, Juan-José. "Estamos locos”. El país, 17 dic. 2010. http://www.elpais.com/articulo/ultima/Estamos/locos/ elpepiult/20101217elpepiult_1/Tes 
Ministerio de Cultura. Observatorio de la lectura y del libro. Boletín n. 3, 2010.

http://www.librerosmadrid.es/files/BOLL_3_Diciembre[1]. $p d f$

Observatorio del libro y la lectura. El libro electrónico, 2010. http://www.mcu.es/libro/docs/MC/Observatorio/pdf/ LIBRO_ELECTRONICO_2010.pdf

Oberholzer, Felix; Strumpf, Koleman. "The effect of file sharing on record sales. An empirical analysis". Journal of political economy, 2007, v. 115, pp. 1-42.

http://www.unc.edu/ cigar/papers/FileSharing_March 2004.pdf

OCDE. Piracy of digital content, 2009.

http://browse.oecdbookshop.org/oecd/pdfs/browseit/ 9309061E.PDF

O'Leary, Brian. Impact of $p 2 p$ and free distribution on book sales. New York: O’Reilly Media, 2009.

Peitz, Martin; Waelbroeck, Patrick. "The effect of internet piracy on music sales: cross-section evidence". Review of economic research on copyright issues, 2004, v. 1, n. 2, pp 71-79.

http://www.serci.org/docs_1_2/waelbroeck.pdf

Reid, Calvin. "BookExpo America 2009: study calls ebook piracy 'overstated'”. Publishers weekly, May 2009.

Rich, Motoko. "Print books are target of pirates on the Web". The New York times, 11 May 2009. http://www.nytimes.com/2009/05/12/technology/internet/ 12digital.html

Rob, Rafael; Waldfogel, Joel. "Piracy on the high C's: music downloading, sales displacement, and social welfare in a sample of college students". Journal of law and economics, 2006, v. XLIX.

http://bpp.wharton.upenn.edu/waldfogj/jle_piracy.pdf

Schulze, Hendrik; Mochalski, Klaus. Internet study 2008/2009. Ipoque, 2009.

http://www.ipoque.com/resources/internet-studies/internetstudy-2008_2009

Stern, David. "Ebooks: from institutional to consortial considerations". Online, 2010, v. 34, pp. 29-36.

Tera Consultants. Building a digital economy: the importance of saving jobs in the EU's creative industries, 2010.

http://www.teraconsultants.fr/assets/publications/PDF/ 2010-Mars-Etude_Piratage_TERA_full_report-En.pdf

TNO, SEO, IViR. Ups and downs. Economic and cultural effects of file sharing on music, film and games, 2009.

http://www.governo.it/Presidenza/antipirateria/audizioni/ audizione_ALTROCONSUMO_allegato2.pdf

Van-Eijk Nico; Poort, Joost; Rutten, Paul. "Legal, economic and cultural aspects of file sharing". Communication and strategies, 2010, n. 1, pp. 35-54.

http://www.ivir.nl/publications/vaneijk/Communications\& Strategies_2010.pdf

\section{El mundo de las Ciencias Sociales está entrelazado y la investigación se extiende a} numerosas disciplinas: desde la Economía a la Política, pasando por la Criminología, la Educación, Ios Servicios Sociales, la Geografía Humana, etc.

\section{Por esta razón, ¿cómo puede estar seguro de que su biblioteca está lo \\ suficientemente preparada para satisfacer las necesidades de los investigadores de las diferentes facultades de su institución?}

Solicite hoy mismo información a su contacto en ProQuest sobre nuestro Paquete Social Sciences Premium.

La colección actual más extensa de productos de Ciencias Sociales, con resúmenes e índices (A\&l) y con texto completo del mercado, en la cual se incluyen:

\section{Fuentes de reconocida solvencia.}

Gran variedad de fuentes internacionales.

Amplia gama de contenido.

Investigaciones directamente relevantes para las ciencias sociales.
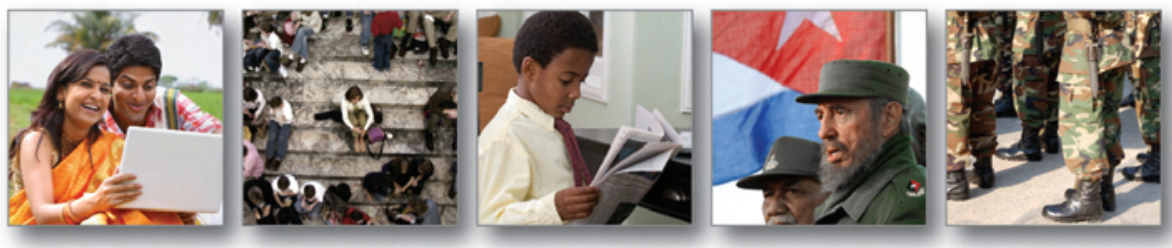

El Paquete Premium contiene las siguientes bases de datos:

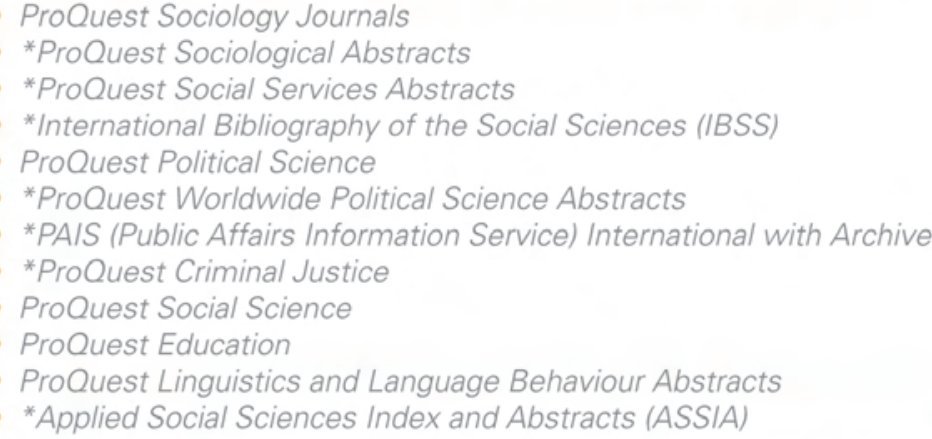

*Con la NUEVA función deep-indexing ayuda a los investigadores a recuperar información que anteriormente era difícil de encontrar (como mapas, tablas, gráficos y cifras) en los resultados de búsqueda. 